\title{
PHARMACOLOGICAL AND BIOPHARMACEUTICAL STUDIES OF PARACETAMOL AND N-ACETYL-D-GLUCOSAMINE COMBINATION AS AN ANALGETIC DRUG
}

\author{
Olena Ruban, Ihor Zupanets, Tetiana Kolisnyk, Sergii Shebeko, Olga Vashchenko, \\ Stanislav Zimin, Olena Dolzhykova
}

\begin{abstract}
The aim. Experimental selection of paracetamol and N-acetyl-D-glucosamine (NAG) ratio and combined dose and bioavailability study of the pharmaceutical composition and active pharmaceutical ingredient (API) in the model of cell biomembranes.

Materials and methods. The following substances were used: paracetamol, Actimask Acetaminophen (gelatin-coated paracetamol), and NAG. For pharmacological studies were used laboratory rats, which received test objects intragastrically. The study was performed on the model of inflammatory hyperalgesia according to the Randall-Selitto method. The values of pain threshold before and after pathology induction were established. For biopharmaceutical studies,

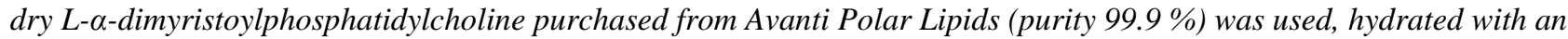
appropriate amount of double-distilled water to obtain $70 \%$ wt./wt. water dispersion. Differential scanning calorimetry (DSC) studies were performed employing DSC 1 microcalorimeter (Mettler Toledo). Pharmaceuticals investigated were placed on the bottom of a crucible, then a proper amount of the lipid membrane was added and this moment was taken as the time reference point $(t=0)$. Then a crucible was sealed with a lid and a sample was undergone consecutive temperature scans, heating from 0 to $35^{\circ} \mathrm{C}$ at a scanning rate of $2{ }^{\circ} \mathrm{C} / \mathrm{min}$. The procedure was repeated until no more changes in DSC profiles were observed, i.e. system equilibrium was reached.

Results. It has been experimentally proven that the combination of paracetamol and NAG in the ratio of 4:1 showed better analgesic efficacy. The dose of active ingredients was determined to be $50 \mathrm{mg} / \mathrm{kg}$ by the sum of API. The active interaction of paracetamol with the bilayer of biomembranes was established and it was determined that Actimask has a worse rate of penetration into the membrane due to the coating of paracetamol with a gelatin shell. NAG didn't significantly affect the rate of penetration of Actimask through the bilayer of membranes, but the auxiliary components of the tablet mixture significantly improved the rate and completeness of penetration of paracetamol through the bilayer of biomembranes.
\end{abstract}

Conclusions. The study found the most effective ratio between paracetamol and NAG in the composition, which is 4:1. The next step was to determine the dose of API, which is $50 \mathrm{mg} / \mathrm{kg}$ of the sum of active substances. It has been determined that paracetamol has good permeability through the bilayer of biomembranes, and the tablet mass significantly improves the permeability of paracetamol

Keywords: paracetamol, $N$-acetyl-D-glucosamine, tablet, rats, analgesics, L- $\alpha$-dimyristoylphosphatidylcholine (DMPC), differential scanning calorimetry (DSC), lipid membrane

\section{How to cite:}

Ruban, O., Zupanets, I., Kolisnyk, T., Shebeko, S., Vashchenko, O., Zimin, S., Dolzhykova, O. (2022). Pharmacological and biopharmaceutical studies of paracetamol and n-acetyl-d-glucosamine combination as an analgetic drug. ScienceRise: Pharmaceutical Science, 1 (35), 28-36. doi: http://doi.org/10.15587/1729-4061.2022.253474

(C) The Author(s) 2022

This is an open access article under the Creative Commons CC BY license hydrate

\section{Introduction}

According to current data, chronic pain (CP) is experienced by a significant proportion of the population in need of long-term analgesic therapy. According to international and national protocols for the treatment of $\mathrm{CP}$, first-line drugs are paracetamol, metamizole sodium, ibuprofen, and other non-steroidal anti-inflammatory drugs (NSAIDs) [1].

The International Association for the Study of Pain (IASP) defines pain as "an unpleasant sensory and emotional sensation associated with actual or potential tissue damage". The IASP also deciphers the concept of $\mathrm{CP}$ : "it is pain that lasts beyond the normal recovery period and/or more than three months" [2]. Today, CP is considered not only as a symptom or syndrome but also as a separate nosology. CP can remain after the healing process and be separated from the damage. Some diseases are accompanied by CP. First of all, these are oncological, rheumatological, neurological diseases, etc. [3, 4].

There are several types of pain according to their origin. Nociceptive pain occurs because of tissue damage under the direct influence of a negative agent. Examples 
of such pain are joint and lower back pain, osteoarthritis, sports injuries, and after surgery. One of the types of nociceptive pain is pain caused by inflammation that occurs in soft tissue injuries [5,6].

Neuropathic pain occurs because of damage or malfunction of the somatosensory nervous system. Such pain occurs with injuries and diseases of various parts of the nervous system. It is usually not accompanied by objective physical symptoms. Neuropathic pain is often described as hot, tingling, prickly, and acute. This type of pain usually occurs a few days or weeks after the injury. Sometimes this pain occurs suddenly, for no apparent reason [7].

Paracetamol (PAR), ibuprofen, naproxen, naproxen, diclofenac, indomethacin, ketoprofen, etc. are recommended for the treatment of $\mathrm{CP}$ according to national and international protocols and recommendations [8]. The use of PAR in the treatment of CP is due to the safety of the drug and fewer side effects compared to other NSAIDs (primarily, the lack of gastro- and cardiotoxicity) $[9,10]$.

The mechanism of action of PAR is the action mainly in the central nervous system by inhibiting both isoforms of cyclooxygenase (COX) COX-1, COX-2, and possibly COX-3, which are involved in the synthesis of prostaglandins. Unlike NSAIDs, PAR does not inhibit cyclooxygenase in peripheral tissues and therefore has no peripheral anti-inflammatory effects. The antipyretic properties of PAR are probably due to the direct effect on the thermoregulatory centers of the hypothalamus, which leads to peripheral vasodilation, sweating, and, consequently, heat dissipation $[11,12]$.

PAR is metabolized mainly in the liver, where most are converted to inactive compounds by conjugation with glucuronic acid and sulfuric acid, which are excreted by the kidneys. Only a small amount is excreted unchanged in the urine or oxidized through the liver cytochrome P450 enzyme system (CYP2E1). Metabolism via CYP2E1 produces the toxic metabolite $\mathrm{N}$-acetylp-benzoquinonymine (NAPQI). The toxic effects of PAR are caused by NAPQI and not by PAR or its major metabolites. At therapeutic doses, NAPQI reacts with the sulfhydryl group of glutathione to form a non-toxic conjugate that is also excreted by the kidneys. High doses of PAR could cause glutathione depletion, NAPQI accumulation, and, consequently, hepatotoxicity [13, 14].

To date, there are not PAR-based drugs on the pharmaceutical market of the world and Ukraine, which are intended for long-term therapy of CP. The solution to this problem may be to create a drug that combines PAR and a hepatoprotector.

$\mathrm{N}$-acetyl-D-glucosamine (NAG) is the most interesting agent among the active pharmaceutical ingredients (APIs) that have a hepatoprotective effect and potentiate the analgesic effect of PAR. In the research of Tahia $\mathrm{H}$. Saleem et al. was found a decrease in the hepatotoxicity of PAR at combined application with NAG in therapeutic doses. Hepatocytes of experimental rats had a condition close to the cells of healthy animals in the control group and much better than in the groups receiving PAR. The combination showed a marked decrease in the hepatotoxicity of PAR [15]. According to the literature, glucosamine affects the metabolism of PAR. Glucosamine inhib- its the activity of the enzyme P450, as a result of which the conversion of PAR occurs in other ways and does not form toxic NAPQI. Due to this, the hepatotoxicity of API is reduced [16]. In the dissertation of Tulyakov V., the potentiating effect of analgesic and anti-inflammatory action of the combination of PAR with glucosamine was investigated. The study revealed the interaction between the components of the composition in the form of potentiation and synergism of the analgesic effect of PAR. However, an effective ratio and dosage between PAR and NAG has not been established by Tulyakov V. [17].

Thus, according to the literature, the combination of PAR with NAG has prospects for the creation of a combined drug aimed specifically at the treatment of CP. By combining it is possible to increase the effectiveness of PAR while reducing its toxicity.

The interaction of drugs (ID) with the lipid bilayer of biomembranes is inevitable because the site of drug administration, their penetration into the systemic bloodstream, entering the target cell, metabolism, etc. is accompanied by interaction with many cells.

It is known that the penetration of most APIs into the cell is carried out by passive diffusion through the lipid bilayer of cells. The interaction of ID with the lipid membrane is a mutual process: on the one hand, it affects the pharmacokinetic properties of pharmaceuticals, on the other - changes in the structural and functional properties of the membranes themselves [18].

Recently, to study the interaction of API with cell membranes, model lipid membranes are widely used, which provide valuable information about absorption, toxic properties, opportunities to optimize drug delivery [19].

There is another important aspect of the interaction of pharmaceuticals with membranes. Modern pharmaceutical compositions usually consist of API, which has a therapeutic effect, as well as a set of excipients that are part of the pharmaceutical composition, which often affect the pharmaceutical, pharmacological, biopharmaceutical performance, and the like.

Therefore, we conducted an experimental study of the interaction of PAR, glucosamine, and tablet mixture with the bilayer of biomembranes with the establishment of the effect of each API and the mixture on the membranes to determine the biopharmaceutical characteristics of the studied pharmaceutical composition.

The study aimed to experimentally select the ratio and dose of the combination of paracetamol and $\mathrm{N}$ acetyl-D-glucosamine and study the bioavailability of the pharmaceutical composition and API on the model of cell biomembranes.

\section{Planning (methodology) of research}

The study of pharmacological and biopharmaceutical characteristics of API and pharmaceutical composition aims to study the analgesic activity of the combination, the interaction at the level of biomembranes, and its prospects for use in pharmaceutical development. To do this, it was necessary to define certain parameters:

a) the effective ratio between PAR and NAG;

b) dose of API by the sum of active substances in the ratio established at the previous stage;

c) interaction of PAR, Actimask Acetaminophen, NAG and the developed pharmaceutical composition 
with biomembranes and possible influence of individual components on the biopharmaceutical parameters of the pharmaceutical mixture.

The study consists of theoretical and practical justification of the amount of API in the development of pharmaceutical compositions and the study of the interaction of API and composition with biomembranes.

\section{Materials and methods}

\subsection{Materials}

Paracetamol (Anqiu Lu`an Pharmaceutical Co., China), gelatin-coated paracetamol (Actimask ${ }^{\circledR}$ Acetaminophen, SPI Pharma, USA), N-acetyl-D-glucosamine (Zhejiang Candorly Pharmaceutical, China), $\lambda$ carrageenan (Sigma Aldrich, USA), Plasdone S-630 (ISP, Switzerland), Kolidon CL (BASF, Germany), citric acid (Merck, Germany), sodium bicarbonate (Merck, Germany), Lubripharm (SPI Pharma, USA), Aspartame (Hyet Sweet, France), Orange flavor (Kerry, Italy).

\subsection{Pharmacological research}

Test objects and its preparation

The combinations of PAR and NAG in ratios 2:1, 3:1 and 4:1 were used as the objects of the research. To prepare the PAR/NAG combinations, PAR was used as Actimask $^{\circledR}$ Acetaminophen (SPI Pharma, USA). Test objects were administered to animals in the form of water suspension prepared using a vehicle containing $0.1 \%$ Tween and $0.5 \%$ Cekol after grinding in a mortar and it was mixed by vortex V-1 plus Biosan (Latvia). Test object suspensions were made at such concentrations that all animals received them at the same dose by volume $-10 \mathrm{ml} / \mathrm{kg}$.

Reference object and its preparation

The reference object was PAR under the trade name Actimask ${ }^{\circledR}$ Acetaminophen (SPI Pharma, USA). The test samples of PAR were also administered in the form of suspension prepared with the vehicle similarly to PAR/NAG combinations.

Experimental animals and grouping

Experimental study was performed using 65 random-bred male albino rats weighing 150-220 g, which were obtained from the vivarium of the Educational and Scientific Institute of Applied Pharmacy, National University of Pharmacy (Kharkiv, Ukraine). The rats were housed under conventional laboratory conditions in standard polypropylene cages in a well-ventilated room at $25 \pm 1{ }^{\circ} \mathrm{C}$ and a relative humidity $55 \pm 5 \%$ with a regular $12 \mathrm{~h}$ light / $12 \mathrm{~h}$ dark cycle $[20,21]$. The animals received standard rat diet and water ad libitum [22]. All studies were performed in accordance with Directive 2010/63/EU about meeting laws, regulations and administrative provisions of the EU states concerning protection of animals used for experimental and other scientific purposes [23]. The experimental protocols were approved by the Bioethics Commission of the National University of Pharmacy (Approval No. 2 of 04 November 2019).

The research was conducted in two stages. The purpose of the first stage was to determine the optimal ratio of PAR and NAG in the composition. At this stage, 30 animals were randomly divided into 5 experimental groups $(n=6)$ as follows: group 1 - control pathology (rats with hyperalgesia receiving vehicle); group 2 - rats with hyperalgesia treated with PAR/NAG (2:1) at $100 \mathrm{mg} / \mathrm{kg}$; group 3 - rats with hyperalgesia treated with PAR/NAG (3:1) at $100 \mathrm{mg} / \mathrm{kg}$; group 4 - rats with hyperalgesia treated with PAR/NAG (4:1) at $100 \mathrm{mg} / \mathrm{kg}$; group 5 - rats with hyperalgesia treated with PAR at $100 \mathrm{mg} / \mathrm{kg}$.

At the second stage, we determined the effective dose of the combination with the most optimal ratio of components. There were taken 35 rats, which were randomly divided into 5 experimental groups $(\mathrm{n}=7)$ as follows: group 1 - control pathology (rats with hyperalgesia receiving vehicle); group 2 - rats with hyperalgesia treated with PAR/NAG (4:1) at $25 \mathrm{mg} / \mathrm{kg}$; group 3 - rats with hyperalgesia treated with PAR/NAG (4:1) at $50 \mathrm{mg} / \mathrm{kg}$; group 4 - rats with hyperalgesia treated with PAR/NAG (4:1) at $100 \mathrm{mg} / \mathrm{kg}$; group 5 - rats with hyperalgesia treated with PAR/NAG (4:1) at $150 \mathrm{mg} / \mathrm{kg}$.

\section{Analgesia experiment}

The Randall-Selitto test was used to assess the analgesic effect of the test objects [24] under the development of inflammatory hyperalgesia in rats [25]. At the beginning of the experiment, the baseline of pain threshold (PT) was measured in all rats using the analgesimeter Ugo Basile 37215 by stimulating pain reaction on the right hind paw [26]. Then the model of inflammatory hyperalgesia was induced in the right hind paw in all rats by subplantar injection of $0.1 \mathrm{ml}$ of $1.0 \% \lambda$-carrageenan solution [27]. In 1 hour after pathology induction, all rats received single intragastric administration of tested and reference drugs at corresponding doses. PAR was administered at a dose of $100 \mathrm{mg} / \mathrm{kg}$, which corresponds to the recommended human single dose extrapolated according to the FDA recommendations [28] and also approximately to the median effective dose of PAR in Randall-Selitto assay [25]. PAR/NAG combinations were studied at equivalent doses by the sum of API. At the second stage, combination PAR/NAG (4:1) was administered at doses of $25-150 \mathrm{mg} / \mathrm{kg}$, which overlaps the possible range of its effective doses. Animals of the control group received equivalent amount of vehicle.

Since the time to reach the maximum blood level and, as a consequence, the most pronounced analgesic effect for oral use of PAR averages 30-60 minutes [2930], assessment of the analgesic activity (AA) of test drugs was performed 1 hour after administration. Then AA was calculated by ability of the tested drugs to decrease hyperalgesia level in animals [27]. Thus, AA calculated as a percentage of the level of PT increase in animals in comparison with control group:

$$
\begin{aligned}
& \text { Analgesic activity }= \\
& =\left(\Delta \mathrm{PT}_{\text {control }}-\Delta \mathrm{PT}_{\text {test }}\right) / \Delta \mathrm{PT}_{\text {control }} \times 100 \%
\end{aligned}
$$

where $\Delta \mathrm{PT}_{\text {test }}$ is the percentage difference in rate of $\mathrm{PT}$ in the test group before and after hyperalgesia induction and 
drug administration; $\Delta \mathrm{PT}_{\text {control }}$ is the percentage difference in rate of PT in the control group before and after hyperalgesia induction and drug administration.

The calculation of $\Delta \mathrm{PT}$ was performed according to the formula (2):

$$
\Delta \mathrm{PT}=\left(\mathrm{PT}_{\text {initial }}-\mathrm{PT}_{\text {final }}\right) / \mathrm{PT}_{\text {initial }} \times 100, \%,
$$

where $\Delta \mathrm{PT}$ - the difference between the initial and final pain threshold; $\mathrm{PT}_{\text {initial }}$ - initial pain threshold; $\mathrm{PT}_{\text {final }}-$ final pain threshold measured 1 hour after drug administration (2 hours after carrageenan administration).

\section{Statistical analysis}

All the results were processed by descriptive statistics and presented as the mean \pm standard error of the mean. Statistical differences between groups were analyzed using one-way ANOVA followed by Tukey's posthoc test [31]. Utilized computer software included IBM SPSS Statistics v. 22 (IBM Corp., USA) and MS Excel 2016 (Microsoft Corp., USA). The level of statistical significance was considered as $\mathrm{p}<0.05$.

\subsection{Lipid membrane preparation}

Dry L- $\alpha$-dimyristoylphosphatidylcholine (DMPC) purchased from Avanti Polar Lipids (purity 99.9\%) was hydrated by a proper amount of bidistilled water to obtain $70 \% \mathrm{wt} / \mathrm{wt}$ water dispersion. During the preparation procedure, the samples were stored at $5{ }^{\circ} \mathrm{C}$ for 3-4 days with regular agitation and heating to $50{ }^{\circ} \mathrm{C}$. Water con- tent was controlled using Mettler XP 26 microbalance (Mettler Toledo).

\subsection{Differential scanning calorimetry}

Differential scanning calorimetry (DSC) studies were performed employing DSC 1 microcalorimeter (Mettler Toledo) according to procedure described in [32]. The samples (10-20 mg) were treated using $40 \mu \mathrm{l}$ standard aluminium crucibles in consecutive temperature scans (heating from 0 to $35^{\circ} \mathrm{C}$ at scanning rate $2{ }^{\circ} \mathrm{C} / \mathrm{min}$ ). Paracetamol content was kept $6 \% \mathrm{wt} / \mathrm{wt}$ relatively to the lipid membrane.

The data obtained were processed with Stare SW 11.0 software. Normalized DSC thermograms were plotted, where original heat flow values were normalized by sample mass values. Sample mass was fixed before and after each DSC experiment employing Mettler XP 26 microbalance (Mettler Toledo). Between the scans, the samples were stored at room conditions.

\section{Results}

According to the results shown in Tab. 1, the development of inflammation was present in all studied animals. After administration of the pharmaceutical compositions, a decrease in limb edema was visually observed in all experimental groups, but with different strengths of onset of effect. This observation confirms the presence of pharmacological activity of the combination in the model of inflammatory hyperalgesia.

Table 1

Results of the assessment of paracetamol and N-acetyl-D-glucosamine ratio in the composition

\begin{tabular}{|c|c|c|c|c|}
\hline \multirow{2}{*}{ Group of animals } & \multicolumn{2}{|c|}{ PT, $\mathrm{g}$} & \multirow{2}{*}{$\Delta \mathrm{PT}, \mathrm{g}$} & \multirow{2}{*}{ AA, $\%$} \\
\hline & initial & final & & \\
\hline Control pathology & $119.4 \pm 13.3$ & $56.1 \pm 9.0$ & $52.1 \pm 2.5$ & - \\
\hline PAR/NAG $(2: 1)$ & $174.4 \pm 20.6$ & $142.0 \pm 13.8$ & $22.2 \pm 2.9 *$ & $57.4 \pm 5.5$ \\
\hline PAR/NAG (3:1) & $150.3 \pm 15.0$ & $113.1 \pm 12.4$ & $24.8 \pm 2.4^{*}$ & $52.3 \pm 4.6$ \\
\hline PAR/NAG (4:1) & $175.7 \pm 14.5$ & $140.3 \pm 12.7$ & $20.0 \pm 4.1 *$ & $61.6 \pm 3.4 * *$ \\
\hline PAR & $166.5 \pm 13.5$ & $118.9 \pm 7.7$ & $27.9 \pm 2.6^{*}$ & $46.6 \pm 3.4$ \\
\hline
\end{tabular}

Note: NAG - N-acetyl-D-glucosamine; PAR - paracetamol; PT-pain threshold; AA - analgesic activity; $* P<0.05$ compared to control pathology group; ** $P<0.05$ compared to group treated with PAR

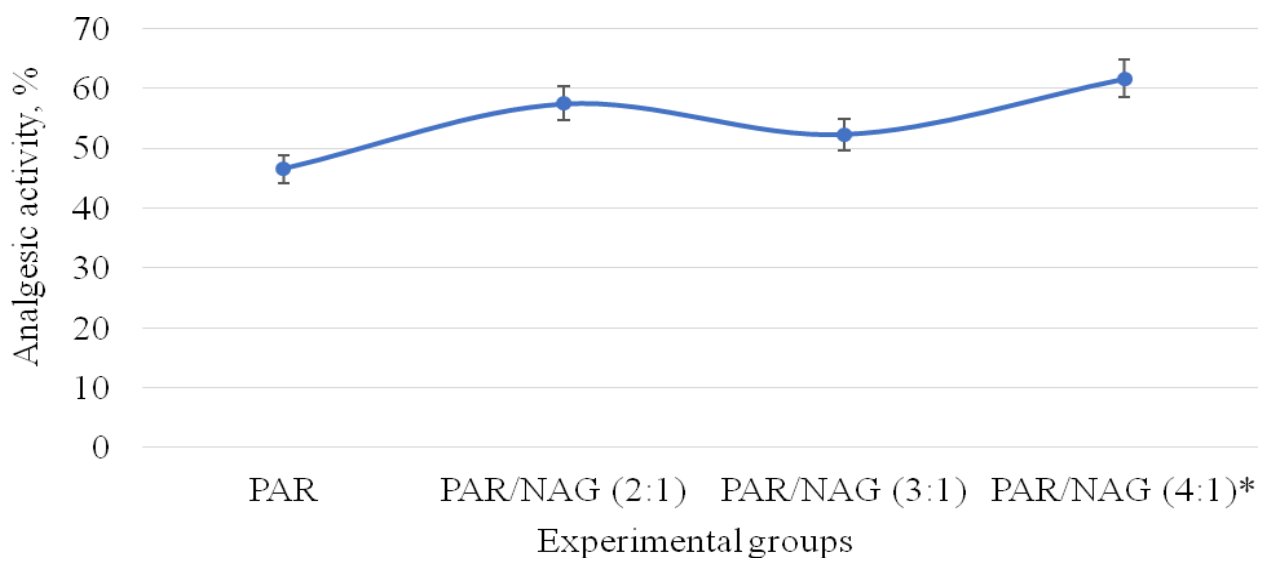

Fig. 1. The curve of changes in the analgesic activity of different ratios of paracetamol and N-acetyl-D-glucosamine in the composition: NAG - N-acetyl-D-glucosamine; PAR - paracetamol; * P $<0.05$ compared to group treated with PAR

As could be seen from Fig. 1, PAR/NAG compositions showed different analgesic effects, but all variants were more active than pure PAR (comparison drug).
However, there is a decrease in the analgesic effect in the option of 3:1, which may indicate the nonlinearity of the indicators. The most pronounced ratio of NAG and PAR 
in terms of hyperalgesia is $4: 1$ by the sum of API, which is statistically significant for paracetamol. Subsequently, this ratio was chosen to find the effective dose. The second stage of the study aimed to determine the most effective total dose of the PAR/NAG combination in a certain ratio of $4: 1$.

The methodology of the study was the same as determining the ratio.

Table 2

Results of the study of the effective doses of the paracetamol and N-acetyl-D-glucosamine combination in a ratio of 4:1

\begin{tabular}{|l|c|c|c|c|}
\hline \multirow{2}{*}{ Group of animals } & \multicolumn{2}{|c|}{ PT, } & \multirow{2}{*}{$\Delta$ PT, $g$} & \multirow{2}{*}{ AA, $\%$} \\
\cline { 2 - 4 } & initial & final & & \\
\hline Control pathology & $236.4 \pm 25.2$ & $82.9 \pm 7.1$ & $65.0 \pm 4.6$ & - \\
\hline PAR/NAG $(4: 1) 25 \mathrm{mg} / \mathrm{kg}$ & $177.1 \pm 18.9$ & $95.0 \pm 11.6$ & $41.6 \pm 8.9$ & $36.0 \pm 9.4$ \\
\hline PAR/NAG $(4: 1) 50 \mathrm{mg} / \mathrm{kg}$ & $217.9 \pm 6.4$ & $186.4 \pm 15.5$ & $15.9 \pm 3.9^{*}$ & $75.6 \pm 6.0^{* *}$ \\
\hline PAR/NAG $(4: 1) 100 \mathrm{mg} / \mathrm{kg}$ & $188.6 \pm 26.6$ & $155.0 \pm 17.2$ & $15.5 \pm 5.0^{*}$ & $76.2 \pm 7.7^{* *}$ \\
\hline PAR/NAG $(4: 1) 150 \mathrm{mg} / \mathrm{kg}$ & $112.9 \pm 12.3$ & $93.6 \pm 5.9$ & $14.6 \pm 4.5^{*}$ & $77.5 \pm 6.9^{* *}$ \\
\hline
\end{tabular}

Note: NAG - N-acetyl-D-glucosamine; PAR - paracetamol; $P T$ - pain threshold; AA - analgesic activity; $* P<0.05$ compared to control pathology group; ** $P<0.05$ compared to group treated with PAR/NAG combination (4:1) at a dose of $25 \mathrm{mg} / \mathrm{kg}$

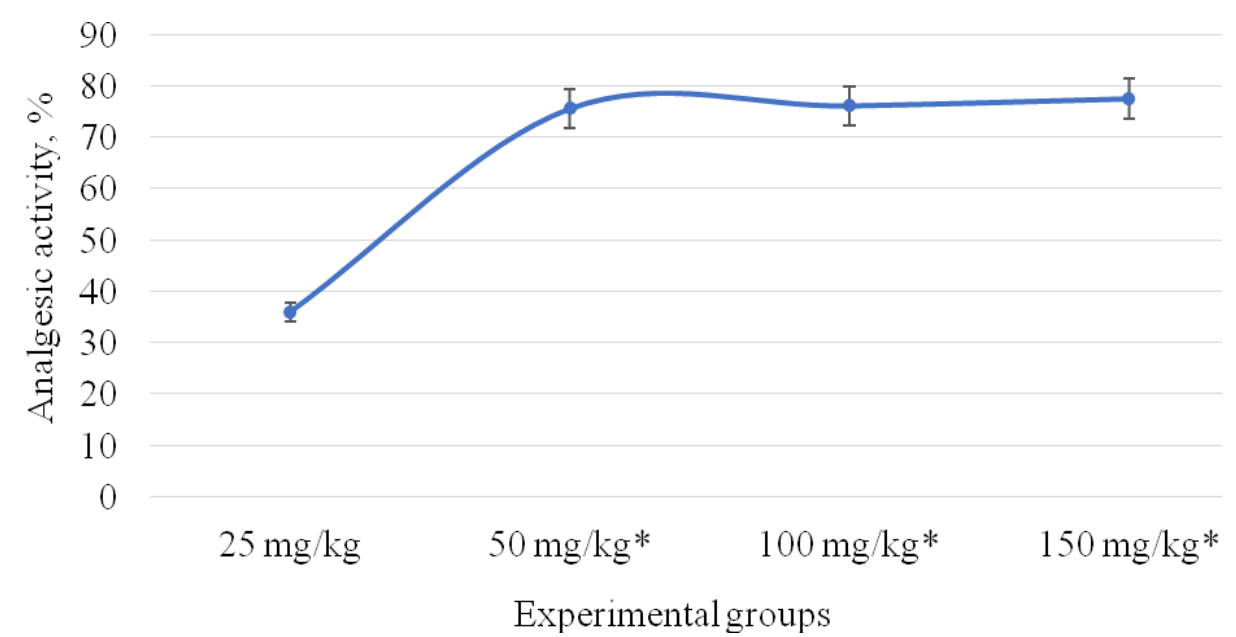

Fig. 2. The curve of changes in the analgesic activity of different doses of the paracetamol and N-acetyl-D-glucosamine combination in a ratio of $4: 1:^{*}-\mathrm{p}<0.05$ compared to group treated with PAR/NAG combination (4:1) at a dose of $25 \mathrm{mg} / \mathrm{kg}$

All studied doses of PAR/NAG combination (4:1) showed analgesic activity in a model of inflammatory hyperalgesia. As could be seen from Table 2 and Fig. 2, there is an increase in activity with increasing dose from $25 \mathrm{mg} / \mathrm{kg}$ to $50 \mathrm{mg} / \mathrm{kg}$. However, after a dose of $50 \mathrm{mg} / \mathrm{kg}$ analgesic activity reaches its "plateau of activity", i.e., doses of $100 \mathrm{mg} / \mathrm{kg}$ and $150 \mathrm{mg} / \mathrm{kg}$ showed the effectiveness of slightly more than $50 \mathrm{mg} / \mathrm{kg}$, which is not statistically significant. It could be concluded that from the point of view of safety-efficacy the most appropriate dose of PAR/NAG combination (4:1) is $50 \mathrm{mg} / \mathrm{kg}$ by the sum of API.

The next component of our research was the study of the interaction of API and tablet mixture with the biomembrane bilayer.

The obtained cascades of DSC profiles are represented in Fig. 3. Fig. 4 is plotted to refer to DSC profiles obtained for the samples at various stages of equilibration. The profile of a neat DMPC membrane contains two endothermic peaks. They correspond to $1^{\text {st }}$ order phase transitions "gel phase $\rightarrow$ ripple phase" $\left(\mathrm{T}_{\mathrm{p}}=15.8^{\circ} \mathrm{C}\right)$ and "ripple phase $\rightarrow$ liquid crystalline phase" $\left(\mathrm{T}_{\mathrm{m}}=24.4^{\circ} \mathrm{C}\right)$ [33]. Upon addition PAR in various combinations, the first peak becomes smeared and disappears in the first few scans.

One should note that each sample demonstrates the unique pattern of DSC profiles which reflects kinetics of drug-lipid interaction [32]. Indeed, for individual PAR (Fig. 3, a), a new low-temperature peak appears at the very first scan. Its position is unchanged almost until equilibrium, but its intensity rises significantly. At the same time, the high-temperature portion of the initial peak drops rather fast and coalesce with the lowtemperature one in $1.7 \mathrm{hrs}$ after the start of the experiment. In the samples containing Actimask (Fig. 3, b), this coalescence takes place much later, after ab. $30 \mathrm{hrs}$. Similar equilibrium values $\mathrm{T}_{\mathrm{m}}{ }^{\mathrm{eq}}=21.7 \pm 0.1{ }^{\circ} \mathrm{C}$ were obtained for the samples containing both Actimask and PAR, as it clears from Fig. 4, b.

The presence of glucosamine (Fig. 3, c) shortens coalescence time to $23 \mathrm{hrs}$ and elevates $\mathrm{T}_{\mathrm{m}}{ }^{\mathrm{eq}}$ value to $22.3^{\circ} \mathrm{C}$. Tablet-mass introduction into the membrane gives a more complex pattern of DSC profiles (Fig. 3, c), but one could specify its pronounced lowering effect on $\mathrm{T}_{\mathrm{m}}{ }^{\text {eq }}$ (to $20.8^{\circ} \mathrm{C}$ ). A control sample was a neat DMPC membrane that remained unchanged all the time (Fig. 4, b). 


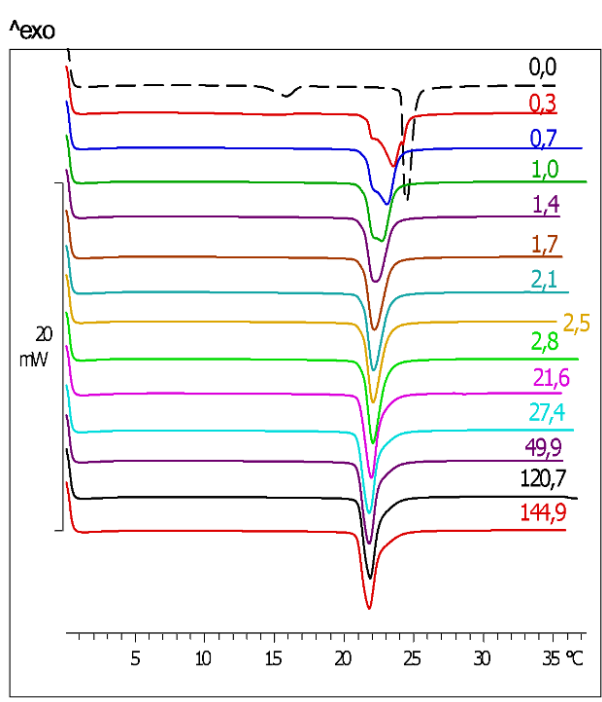

Lab: METILER

$a$

^exo

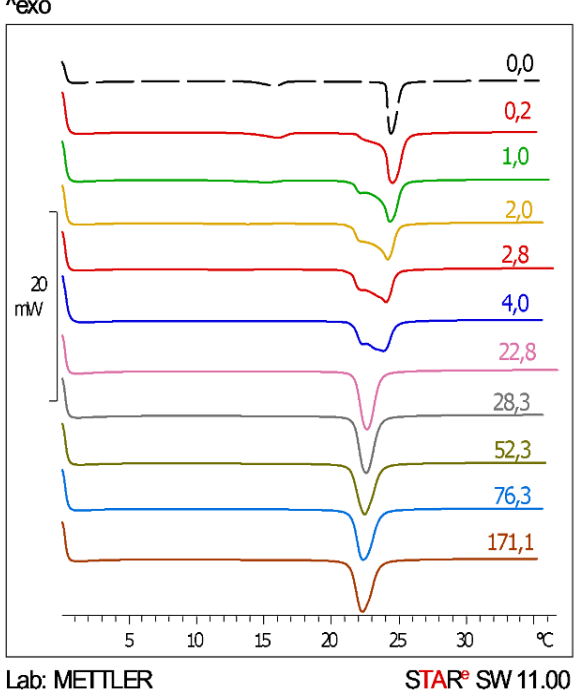

$c$ ^exo

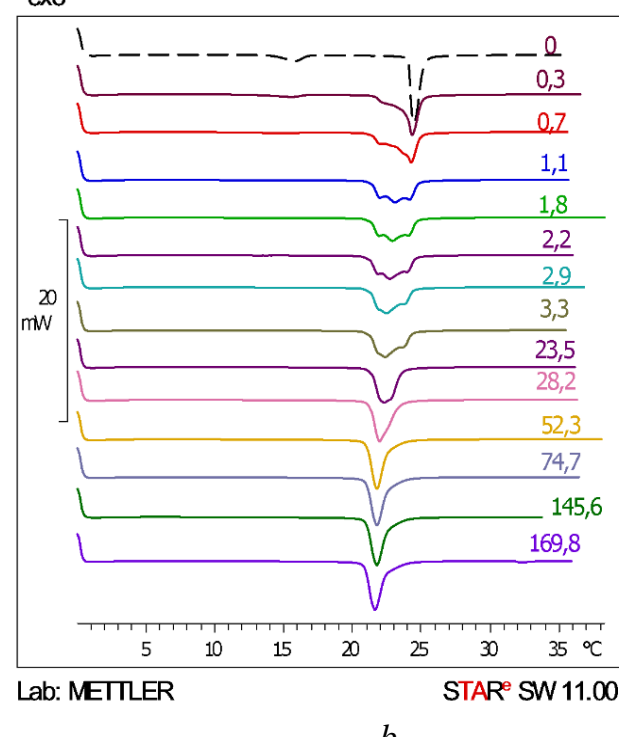

^exo

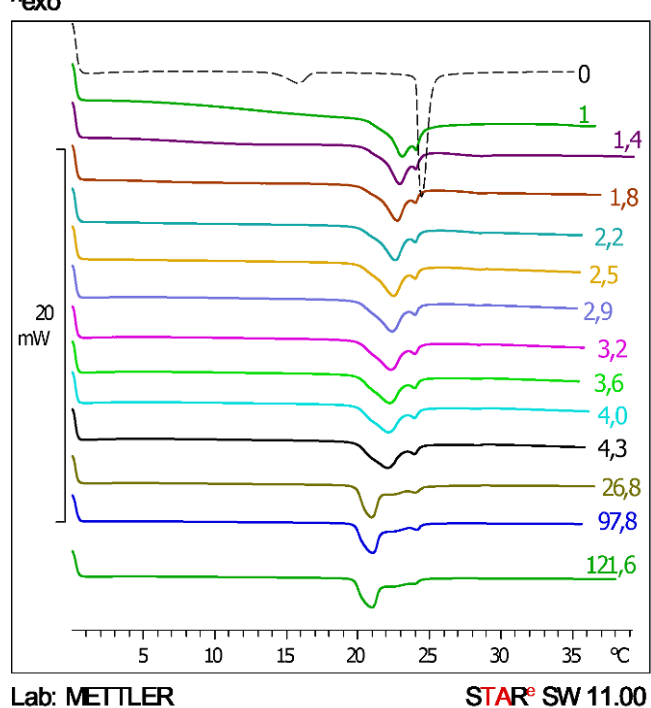

$d$

Fig. 3. DSC profiles of lipid membranes containing: $a$ - paracetamol; $b$ - Actimask; $c$ - Actimask + glucosamine; $d$-tablet-mass. The numbers on the right of the curves indicate the time (in hrs) since the start of the experiment. The DSC profile of the neat lipid membrane (dotted line) corresponds to the time reference point $(\mathrm{t}=0)$

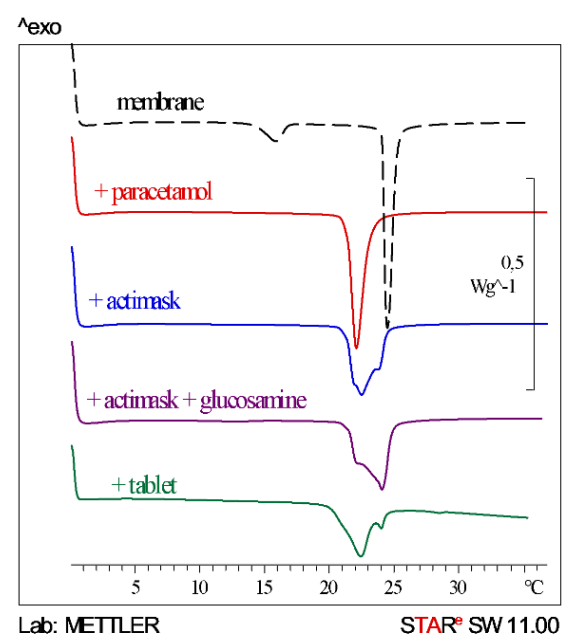

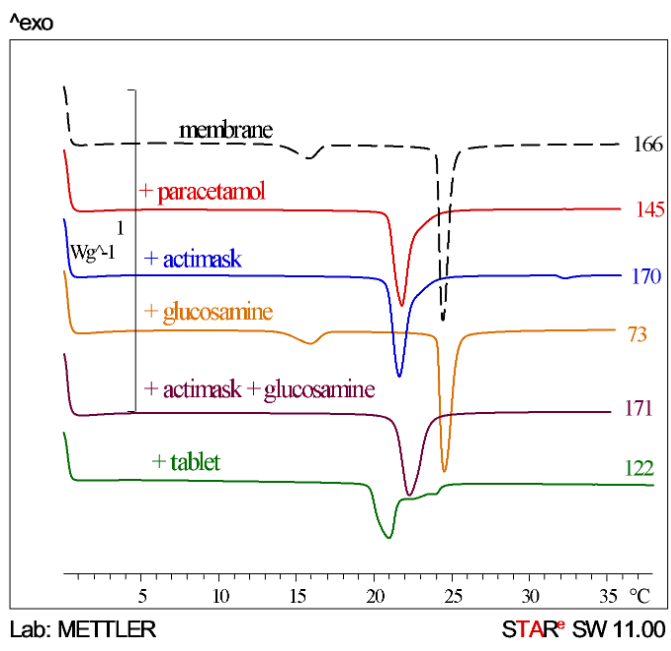

$b$

Fig. 4. Normalized DSC profiles of lipid membranes containing pharmaceuticals: $a$ - after 3 hrs since the start of experiments; $b$-after systems equilibrium (time (hrs) is indicated in numbers on the right of the curves). DSC profiles of the neat lipid membrane are marked with dotted lines 


\section{Discussion}

As a result of our work, there is a confirmation of previous studies presented in the dissertation of Tulyakov V., where the potentiating effect of the analgesic combination of PAR with glucosamine was given [17]. An increase in the analgesic activity of the combination of PAR as Actimask with NAG was found in comparison with the PAR mono-preparation.

As we could see from the results of the study (Table 1 and Fig. 1), the analgesic activity of the PAR monopreparation is weaker than in the composition. At a lower dose of PAR, the effectiveness of the drug in a mixture with NAG is much higher (by $15 \%$ when comparing the mono-preparation of PAR and the composition 4: 1). However, there is a decrease in the analgesic effect in the option of 3: 1, which may indicate the nonlinearity of the indicators. The most analgesically active ratio of PAR and NAG is 4: 1 . Subsequently, this ratio was chosen to find the effective dose.

All combinations showed analgesic activity in a model of carrageenan inflammatory hyperalgesia. As could be seen from Table 2 and Fig. 2, there is an increase in activity between doses of $25 \mathrm{mg} / \mathrm{kg}$ and $50 \mathrm{mg} / \mathrm{kg}$. However, after a dose of $50 \mathrm{mg} / \mathrm{kg}$ analgesic activity reached its "plateau of activity", i.e., doses of $100 \mathrm{mg} / \mathrm{kg}$ and $150 \mathrm{mg} / \mathrm{kg}$ showed the efficacy of slightly more than $50 \mathrm{mg} / \mathrm{kg}$ and are statistically insignificant. It could be concluded that from the point of view of safety-efficacy, i.e., further increase in dosage does not increase efficiency, however, it is possible to detect side effects of PAR, the most appropriate dose is $50 \mathrm{mg} / \mathrm{kg}$ by the sum of active substances PAR and NAG in a ratio of $4: 1$. Therefore, for further studies, we chose the lowest dose without loss of analgesic activity, but with less likely side effects.

It is worth noting that model lipid membranes used in the present study could be represented as ordered planar multibilayer structures where lipid bilayers are separated one from another by water phase. Kinetics of drug-membrane interactions could be affected by a set of processes: drug release, drug sorption by a lipid bilayer, drug diffusion through lipid/water stacks, etc. The presence of additional compounds could impact all these processes as well to shift equilibrium drug distribution between water and lipid phases. Indeed, there is a wide diversity of joint membranotropic effects caused both by API combinations [34] and by excipients in drug formulations [35]. Based on the above, we could interpret the results obtained in biopharmaceutical experiments.

As one could see from Fig. 4, a, PAR and Actimask differ substantially in the kinetics of drugmembrane interactions, and the most probable limitation stage seems drug release from gelatin capsules. Joint administration of Actimask and glucosamine to the lipid membrane hinders PAR diffusion through lipid bilayers as it is clear from a significant portion of undisturbed DSC peak. Meanwhile, tablet-mass demonstrates improved kinetics even relative to individual PAR, which is caused by joint drug-excipient membranotropic effects.

Equilibrium state (Fig. 4, b) demonstrate that gelatin does not impact PAR distribution into lipid membrane and localizes in distinct membrane regions (see small high-temperature peak on "Actimask" profile). Glucosamine individually induces practically no changes in membrane ordering (both initial DMPC peaks, $\mathrm{T}_{\mathrm{m}}$ and $\mathrm{T}_{\mathrm{p}}$, remain undisturbed), but reduces PAR distribution which reflects in $\mathrm{T}_{\mathrm{m}}{ }^{\mathrm{eq}}$ elevation on "Actimask + glucosamine" profile. Finally, tablet-mass causes sufficient $\mathrm{T}_{\mathrm{m}}{ }^{\text {eq }}$ diminishing which bears witness to the promotion of PAR distribution to the membrane.

Taken together, all our findings point that the drug formulation improves both kinetics and equilibrium characteristics of PAR -membrane interactions.

Study limitations. Certain limitations in the work used in the method of studying the analgesic activity of the drug. The method of inflammatory analgesia characterizes the action of analgesics during the development of edema and inflammation. However, chronic pain is not always caused by inflammation and there are other mechanisms of pain response. There are also restrictions on the use of only the biomembrane bilayer model, which contains only one type of phospholipid.

Prospects for further research. There is interest in the study of analgesic activity in other pharmacological models. Research on a wider range of lipid bilayers of biomembranes. The prospect of further research is also to study the hepatoprotective and toxic effects of the pharmaceutical combination in subchronic modeling of the drug.

\section{Conclusions}

The study found the most effective ratio between PAR as Actimask and NAG, which is 4:1. The effective dose in the selected ratio becomes constant at $50 \mathrm{mg} / \mathrm{kg}$, and a further increase in dosage does not show an increase in effectiveness, but it is possible to detect side effects of PAR. Therefore, for further studies, we chose the lowest dose without loss of analgesic activity, but with less likely side effects.

The active interaction of PAR with the bilayer of biomembranes was found and it was determined that the coating with a gelatin shell (substance Actimask Acetaminophen) impairs the rate of penetration of API into the membrane. NAG did not significantly affect the rate of penetration of Actimask Acetaminophen through the bilayer of membranes, but the auxiliary components of the tablet mixture significantly improved the rate and completeness of penetration of API through the bilayer of biomembranes.

Thus, the studied tool has prospects for analgesia of inflammatory acute pain with predicted hepatoprotective activity, which is also a considerable advantage in the case of long-term use. The improvement of biopharmaceutical parameters of the drug in the study of API penetration through the bilayer of membranes due to the auxiliary components of the developed pharmaceutical composition was determined.

\section{Conflict of interests} interests.

The authors declare that they have no conflict of

\section{Financing} support. 


\section{References}

1. Zupanets, I. V., Ruban, O. A., Kolisnyk, T. E. (2019). The modern state of orally disintegrating tablets development. Ukrainian biopharmaceutical journal, 3 (60), 6-12. doi: http://doi.org/10.24959/170227

2. International Association for the Study of Pain (2021). International Association for the Study of Pain. Available at: https://www.iasp-pain.org/

3. Rachin, A., Sharov, M., Averchenkova, A., Vygovskaya, S., Nuvakhova, M. (2017). Chronic pain: from pathogenesis to innovative treatment. RMJ, 9, 625-631.

4. International Association for the Study of Pain (2021). IASP News. Available at: https://www.iasp-pain.org/ publications/iaspnews/?ItemNumber=8340\&navItemNumber $=643$

5. Takai, Y., Yamamoto-Mitani, N., Abe, Y., Suzuki, M. (2014). Literature review of pain management for people with chronic pain. Japan Journal of Nursing Science, 12 (3), 167-183. doi: http://doi.org/10.1111/jjns.12065

6. Chronic pain: a literature review (2021). Saúde Coletiva: Uma Abordagem Multidisciplinar 3, 38-45.

7. Sulik, R. V. (2016). Differential approach in the diagnosis and treatment of neuropathic pain. Pain Medicine Journal, 1, 51-56.

8. Zupanets, I. V., Ruban, O. A., Ievtushenko, O. M., Kolisnyk, T. E. (2020). Assortment research of analgesic drugs for chronic pain treatment on pharmaceutical market of Ukraine. Farmatsevtychnyi Zhurnal, 3, 16-28. doi: http://doi.org/10.32352/0367-3057.3.20.02

9. Chronic pain: supporting safer prescribing of analgesics (2017). British Medical Association, 35.

10. Nikles, C. J., Yelland, M., Del Mar, C., Wilkinson, D. (2005). The Role of Paracetamol in Chronic Pain: An EvidenceBased Approach. American Journal of Therapeutics, 12 (1), 80-91. doi: http://doi.org/10.1097/00045391-200501000-00011

11. The International Agency for Research on Cancer. (1999). IARC Monographs on the Evaluation of Carcinogenic Risk to Humans Vol. 73. Paracetamol. World Health Organization.

12. Viktorov, A. P. (2010). Acetaminophen (paracetamol) - non-steroidal anti-inflammatory analgesic-antipyretic. Rational Pharmacotherapy, 14 (1), 42-47. Available at: https://rpht.com.ua/ru/archive/2010/1\%2814\%29/pages-42-47/acetaminofen-paracetamolnesteroidnyy-protivovospalitelnyy-analgetik-antipiretik

13. Agrawal, S., Khazaeni, B. (2021). Acetaminophen Toxicity. The National Center for Biotechnology Information. Available at: https://www.ncbi.nlm.nih.gov/books/NBK441917/\#article-17075.s2

14. Farrell, S. E. (2021). Acetaminophen Toxicity: Practice Essentials, Background, Pathophysiology. Medscape. Available at: https://emedicine.medscape.com/article/820200-overview

15. Saleem, T. H., Abo El-Maali, N., Hassan, M. H., Mohamed, N. A., Mostafa, N. A. M., Abdel-Kahaar, E., Tammam, A. S. (2018). Comparative Protective Effects of N-Acetylcysteine, N-Acetyl Methionine, and N-Acetyl Glucosamine against Paracetamol and Phenacetin Therapeutic Doses-Induced Hepatotoxicity in Rats. International Journal of Hepatology, 2018, 1-8. doi: http://doi.org/10.1155/ 2018/7603437

16. Qinna, N. A., Shubbar, M. H., Matalka, K. Z., Al-Jbour, N., Ghattas, M. A., Badwan, A. A. (2015). Glucosamine Enhances Paracetamol Bioavailability by Reducing Its Metabolism. Journal of Pharmaceutical Sciences, 104 (1), 257-265. doi: http://doi.org/10.1002/jps.24269

17. Tulyakov, V. A. (2012). Development of antiarthritic drugs with chondroprotective, anti-inflammatory and analgesic action based on glucosamine amino sugar. NUPh.

18. Sadchenko, A. O., Vashchenko, O. V. et. al. (2017). Some Characteristics of Interactions of Pharmaceuticals and Their Active Pharmaceutical Ingredients with Lipid Membranes. Biophysics, 62 (3), 701-711.

19. Kasian, N. A., Pashynska, V. A., Vashchenko, O. V., Krasnikova, A. O., Gömöry, A., Kosevich, M. V., Lisetski, L. N. (2014). Probing of the combined effect of bisquaternary ammonium antimicrobial agents and acetylsalicylic acid on model phospholipid membranes: differential scanning calorimetry and mass spectrometry studies. Mol. BioSyst., 10 (12), 3155-3162. doi: http://doi.org/10.1039/c4mb00420e

20. Guide for the care and use of laboratory animals (No. 8th) (2011). Washington: National Academies Press.

21. Sharp, P., Villano, J. S. (2012). The Laboratory Rat (Laboratory Animal Pocket Reference). CRC Press, 399. doi: http://doi.org/10.1201/b13862

22. Suckow, M. A., Hankenson, F., Wilson, R. P., Foley, P. L. (2019). American College of Laboratory Animal Medicine. The Laboratory Rat. Academic Press.

23. Directive 2010/63/EU of the European Parliament and of the Council of 22 September 2010 on the protection of animals used for scientific purposes (2010). Official Journal of the European Union.

24. Hasani, A., Soljakova, M., Jakupi, M. H., Ustalar-Ozgen, S. Z. (2011). Preemptive analgesic effect of diclofenac: experimental in rats. Middle East Journal of Anesthesiology, 21 (3), 355-360.

25. Randall, L. O., Selitto, J. J. (1957). A method for measurement of analgesic activity on inflamed tissue. Archives Internationales de Pharmacodynamie, 111 (4), 409-419.

26. Nkeh-Chungag, N. B., Mxolisi Bekwa, P. C., Ndebia, J. E., Kayo, M., Mbafor, T. J., Iputo, E. J. (2010). Analgesic and anti-inflammatory properties of Oxyanthus unilocularis. Journal of Medicinal Plants Research, 4 (10), 932-939.

27. Vogel, H. G. (2007). Analgesic, Anti-inflammatory, and Anti-pyretic Activity. Drug Discovery and Evaluation. Berlin, Heidelberg: Springer, 983-1116. doi: https//doi.org/10.1007/978-3-540-70995-4_9

28. Guidance for industry: Estimating the Maximum Safe Starting Dose in Initial Clinical Trials for Therapeutics in Adult Healthy Volunteers (2005). Food and Drug Administration. Rockville: FDA.

29. Botting, R. M. (2000). Mechanism of Action of Acetaminophen: Is There a Cyclooxygenase 3? Clinical Infectious Diseases, 31 (5), S202-S210. doi: http://doi.org/10.1086/317520

30. Ince, I., Aksoy, M., Ahiskalioglu, A., Comez, M., Dostbil, A., Celik, M. et. al. (2015). A Comparative Investigation of the Analgesic Effects of Metamizole and Paracetamol in Rats. Journal of Investigative Surgery, 28 (3), 173-180. doi: http://doi.org/10.3109/08941939.2014.998798

31. Islam, A. M., Al-Shiha, A. (2018). Foundations of Biostatistics. Springer. doi: http://doi.org/10.1007/978-981-10-8627-4

32. Kasian, N. A., Vashchenko, O. V., Budianska, L. V., Brodskii, R. Y., Lisetski, L. N. (2019). Thermodynamics and kinetics of joint action of antiviral agent tilorone and DMSO on model lipid membranes. Biochimica et Biophysica Acta (BBA) Biomembranes, 1861 (1), 123-129. doi: http://doi.org/10.1016/j.bbamem.2018.08.007

33. Koynova, R., Caffrey, M. (1998). Phases and phase transitions of the phosphatidylcholines. Biochimica et Biophysica Acta (BBA) - Reviews on Biomembranes, 1376 (1), 91-145. doi: http://doi.org/10.1016/s0304-4157(98)00006-9 
34. Lisetski, L. N., Vashchenko, O. V., Kasian, N. A., Sviechnikova, L. V. (2021). Lyotropic Liquid Crystal Phases of Phospholipids as Model Tools in Molecular Biophysics and Pharmacology. Soft Matter Systems for Biomedical Applications. Springer, 85-111. doi: http://doi.org/10.1007/978-3-030-80924-9_4

35. Sadchenko, A. O., Vashchenko, O. V., Puhovkin, A. Y., Kopeika, E. F., Kasian, N. A., Budianska, L. V. et. al. (2017). The characteristics of interactions of pharmaceuticals and their active ingredients with lipid membranes. Biophysics, 62 (4), 570-579. doi: http://doi.org/10.1134/s0006350917040194

Received date 11.01.2021

Accepted date 17.01.2022

Published date 28.02.2022

Olena Ruban*, Doctor of Pharmaceutical Sciences, Professor, Head of Department, Department of Industrial Technology of Drugs, National University of Pharmacy, Pushkinska str., 53, Kharkiv, Ukraine, 61002

Ihor Zupanets, Department of Industrial Technology of Drugs, National University of Pharmacy, Pushkinska str., 53, Kharkiv, Ukraine, 61002

Tetiana Kolisnyk, PhD, Assistant Professor, Department of Industrial Technology of Drugs, National University of Pharmacy, Pushkinska str., 53, Kharkiv, Ukraine, 61002

Sergii Shebeko, Doctor of Pharmaceutical Sciences, Professor, Department of Clinical Pharmacology and Clinical Pharmacy, National University of Pharmacy

Pushkinska str., 53, Kharkiv, Ukraine, 61002

Olga Vashchenko, Doctor of Physical and Mathematical Sciences, Senior Scientist, Leading Researcher, Yu. V. Malyukin Department of Nanostructural Materials, Institute of Scintillation Materials, State Scientific Institution "Institute for Single Crystals" of National Academy of Sciences of Ukraine, Nauky ave., 60, Kharkiv, Ukraine, 61072

Stanislav Zimin, PhD, Associate Professor, Department of Clinical Pharmacology and Clinical Pharmacy, National University of Pharmacy, Pushkinska str., 53, Kharkiv, Ukraine, 61002

Olena Dolzhykova, Doctor of Pharmaceutical Sciences, Associate Professor, Department of Clinical Laboratory Diagnostics, National University of Pharmacy, Pushkinska str., 53, Kharkiv, Ukraine, 61002

*Corresponding author: Olena Ruban,e-mail: ruban_elen@ukr.net 